\title{
Efficacy and safety outcomes of recanalisation procedures in patients with acute symptomatic pulmonary embolism: systematic review and network meta-analysis
}

\author{
David Jimenez, ${ }^{1,2}$ Carlos Martin-Saborido, ${ }^{3}$ Alfonso Muriel, ${ }^{4}$ Javier Zamora, ${ }^{4}$ \\ Raquel Morillo, ${ }^{1,2}$ Deisy Barrios, ${ }^{1,2}$ Frederikus A Klok, ${ }^{5}$ Menno V Huisman, ${ }^{5}$ \\ Victor Tapson, ${ }^{6}$ Roger D Yusen ${ }^{7}$
}

- Additional material is published online only. To view please visit the journal online (http://dx.doi.org/10.1136/ thoraxjnl-2017-210040).

For numbered affiliations see end of article.

Correspondence to Dr David Jimenez, Respiratory Department, Ramón y Cajal Hospital, Madrid 28034, Spain; djimenez.hrc@gmail.com

DJ and CM-S contributed equally.

Received 23 January 2017 Revised 13 October 2017 Accepted 23 October 2017 Published Online First 13 November 2017

Check for updates

To cite: Jimenez $D$, Martin Saborido C, Muriel A, et al.

Thorax 2018:73:464-471.

\section{ABSTRACT}

Background We aimed to review the efficacy and safety of recanalisation procedures for the treatment of PE.

Methods We searched PubMed, the Cochrane Library, EMBASE, EBSCO, Web of Science and CINAHL databases from inception through 31 July 2015 and included randomised clinical trials that compared the effect of a recanalisation procedure versus each other or anticoagulant therapy in patients diagnosed with PE. We used network meta-analysis and multivariate randomeffects meta-regression to estimate pooled differences between each intervention and meta-regression to assess the association between trial characteristics and the reported effects of recanalisation procedures versus anticoagulation.

Results For all-cause mortality, there were no significant differences in event rates between any of the recanalisation procedures and anticoagulant treatment (full-dose thrombolysis: OR 0.60; 95\% CI0.36 to 1.01; low-dose thrombolysis: $0.47 ; 95 \% \mathrm{Cl} 0.14$ to 1.59 ; and catheter-associated thrombolysis: $0.31 ; 95 \% \mathrm{Cl} 0.01$ to 7.96). Full-dose thrombolysis increased the risk of major bleeding $(2.00 ; 95 \% \mathrm{Cl} 1.06$ to 3.78$)$ compared with anticoagulation. Catheter-directed thrombolysis was associated with the lowest probability of dying (surface under the cumulative ranking curve (SUCRA), 0.67), followed by low-dose thrombolysis (SUCRA, 0.66) and full-dose thrombolysis (SUCRA, 0.55). Similarly, low-dose thrombolysis was associated with the lowest probability of major bleeding (SUCRA, 0.61), followed by catheterdirected thrombolysis (SUCRA, 0.54) and full-dose thrombolysis (SUCRA, 0.17). The results were similar in sensitivity analyses based on restricting only to studies in haemodynamically stable patients with PE.

Conclusions In the treatment of $P E$, recanalisation procedures do not seem to offer a clear advantage compared with standard anticoagulation. Low-dose thrombolysis was associated with the lowest probability of dying and bleeding.

Trial registration number PROSPERO CRD42015024670.

\section{INTRODUCTION}

Although most patients with acute PE have an uncomplicated clinical course while undergoing

\section{Key messages}

What is the key question?

- For treatment of acute PE, the benefits and risks of the different recanalisation procedures (ie, full-dose systemic thrombolysis, reduceddose systemic thrombolysis or catheter-directed thrombolysis) versus each other lack clarity.

What is the bottom line?

- Compared with standard anticoagulation, recanalisation procedures had a similar risk of all-cause mortality, and full-dose thrombolysis was associated with an increased risk of major bleeding.

Why read on?

- Low-dose thrombolysis was associated with the lowest probability of dying and bleeding.

standard anticoagulation treatment, the overall short-term mortality rate is still significant. ${ }^{12}$ Death from acute PE usually occurs before or soon after hospital admission. ${ }^{34}$

There have been two main treatments for acute $\mathrm{PE}$, anticoagulant therapy alone or systemic thrombolytic therapy. ${ }^{5}$ Most patients presenting to the hospital with PE have normal blood pressure, normal right ventricular function and a low clinical severity score and therefore have a very low short-term mortality with prompt initiation of anticoagulation. Although systemic thrombolysis has angiographic and haemodynamic benefits for patients with acute PE, compared with standard therapy, it markedly increases major bleeding, including intracranial and fatal bleeding. ${ }^{6}$ Consequently, systemic thrombolytic therapy is usually reserved for patients with PE with haemodynamic instability. ${ }^{7}$ The ability to actively remove emboli in patients with acute PE without increasing bleeding would be an important advance. Low-dose systemic thrombolysis and catheter-based thrombolytic therapy require only a fraction of the systemic fibrinolytic dose, and this dose reduction might improve the safety of thrombolysis for PE. A common problem in evaluating the efficacy of these interventions is the lack of trials (or 
a paucity of available trials) that directly compare these interventions. As a result, no meta-analysis has comprehensively compared the effect of a recanalisation procedure versus each other in patients diagnosed with acute symptomatic PE.

The primary aim of our study was to perform a network meta-analysis of randomised controlled trials (RCTs) for treatment of acute PE to obtain a better estimate of the benefits and risks of the different recanalisation procedures (ie, full-dose systemic thrombolysis, reduced-dose systemic thrombolysis or catheter-directed thrombolysis) versus each other or anticoagulant therapy.

\section{METHODS}

\section{Data sources and searches}

This systematic review is reported according to the Preferred Reporting Items for Systematic Reviews and Meta-analyses statement extension for network meta-analysis and was conducted following an a priori established protocol registered with PROSPERO. ${ }^{8}$ We searched PubMed, the Cochrane Library, EMBASE, EBSCO, Web of Science and CINAHL databases. Each database was searched from its inception date to 31 July 2015. Conference abstracts were included in our search. The retrieved articles were examined to eliminate potential duplicates or overlapping data. No limits or language restriction were applied during the search. The RCTs were identified using the Cochrane Collaboration highly sensitive search strategy (sensitivity-maximising and precision-maximising version). ${ }^{9}$ The search string was: (1) pulmonary embolis*; (2) thrombolysis OR thrombolytic therapy OR streptokinase OR urokinase OR tenecteplase OR alteplase OR desmoteplase OR tissue plasminogen activator OR clot-dissolving medication; (3) search strings 1 AND 2. We also hand searched the references of relevant articles for additional clinical trials not identified by the electronic search and contacted experts. Finally, we searched ClinicalTrials.gov for information on clinical trials that were terminated but unpublished. The planned analysis was registered at the PROSPERO international prospective register of systematic reviews on 20 July 2015 (CRD42015024670).

\section{Study selection}

One reviewer (DJ) performed the database search and initial screening of titles and abstracts. Two investigators (DJ and RM) independently carried out full-text screening of all eligible articles. We included a study if participants were patients with acute symptomatic PE objectively diagnosed with standard imaging techniques and received anticoagulant therapy; the intervention was treatment with a recanalisation procedure (ie, full-dose systemic thrombolysis, reduced-dose systemic thrombolysis or catheter-directed thrombolysis); the comparison group was either treatment with a different recanalisation procedure or no recanalisation treatment (ie, the patients received standard anticoagulation); it was an RCT; and it reported mortality outcomes. Observational studies and trials without a control group were excluded.

\section{Data extraction and quality assessment}

Two reviewers (DJ and RM) independently extracted data onto a computer spreadsheet, with discrepancies resolved by consensus. Extracted data included first author, year of publication, type of intervention and control group, number of patients, patient characteristics and duration of follow-up. The primary outcomes were all-cause mortality and major bleeding, as defined by the study protocol. Secondary outcomes were risk of intracranial haemorrhage (ICH) and recurrent embolism. The occurrence of these outcomes was abstracted according to the intention-totreat population for individual trials. The outcomes data from the first available time point identified as a primary end point from each trial were incorporated into our primary analysis. Each study was graded for potential bias into low, high and unclear according to the Cochrane Collaboration handbook. ${ }^{10}$

\section{Data synthesis and analysis}

Separate meta-analyses of direct evidence only (pairwise meta-analyses) were performed using DerSimonian and Laird random-effects model to estimate pooled ORs and 95\% CIs. ${ }^{11}$ Forest plots were created for each outcome. When there were no events in one treatment group, we used a 0.5 continuity correction. Heterogeneity was assessed using the estimated betweenstudy variance $\left(\tau^{2}\right)$, Cochran $\chi^{2}$ test and the $\mathrm{I}^{2}$ statistic. ${ }^{12}$

Because there are few trials making head-to-head comparisons between recanalisation procedures, we performed a network meta-analysis. Unlike traditional meta-analyses, this method has the advantage of allowing trials comparing recanalisation procedures with some other common treatment (eg, placebo) to be incorporated into the analysis, thus increasing power and enabling a better comparison of recanalisation therapies to be made. ${ }^{13}$ We used multivariate, random-effects meta-regressions to perform each analysis using the network family of commands in Stata. ${ }^{14}$ We evaluated inconsistency between direct and indirect sources of evidence by comparison of the fit and parsimony of consistency and inconsistency models and by calculation of the difference between direct and indirect estimates of a specific treatment effect ('loop-specific approach'). The relative ranking of recanalisation interventions on primary and secondary outcomes was presented as their surface under the cumulative ranking curve (SUCRA) probabilities, which represent their likelihood of being ranked best. ${ }^{15}$ In this study, higher SUCRA scores reflect lower associated all-cause mortality and bleeding events. We estimated the probability of each treatment being the best by averaging 10000 Monte Carlo replications. The level of statistical significance was set at $\mathrm{P}<0.05$ and all statistical tests were 2-sided.

We performed some sensitivity analyses to assess the robustness of the findings. These were based on (1) restricting only to studies in patients with haemodynamically stable PE; (2) restricting only to trials where the mean age of participants in the thrombolytic group was $>65$ years; and (3) alternative statistical model (frequentist approach using a random-effects inconsistency model).

\section{RESULTS}

From a total of 930 unique studies identified using the search strategy, 22 RCTs (2494 patients) were included in the network meta-analysis (online supplementary efigure 1). These included 16 trials comparing full-dose thrombolysis to no thrombolysis (2016 patients), ${ }^{6}{ }^{16-30} 1$ comparing low-dose thrombolysis to no thrombolysis (121 patients), ${ }^{31} 1$ comparing ultrasound-assisted catheter-directed thrombolysis with no thrombolysis $\left(59\right.$ patients) ${ }^{32}$ and 4 comparing full-dose thrombolysis with low-dose thrombolysis (298 patients). ${ }^{33-36}$ The available direct comparisons and network of trials are shown in figure 1 and online supplementary efigures $2-4$.

\section{Characteristics of included studies}

The RCTs included in the network meta-analysis are summarised in table 1. Overall, these 22 trials were reported between 1970 
Low-dose thrombolysis

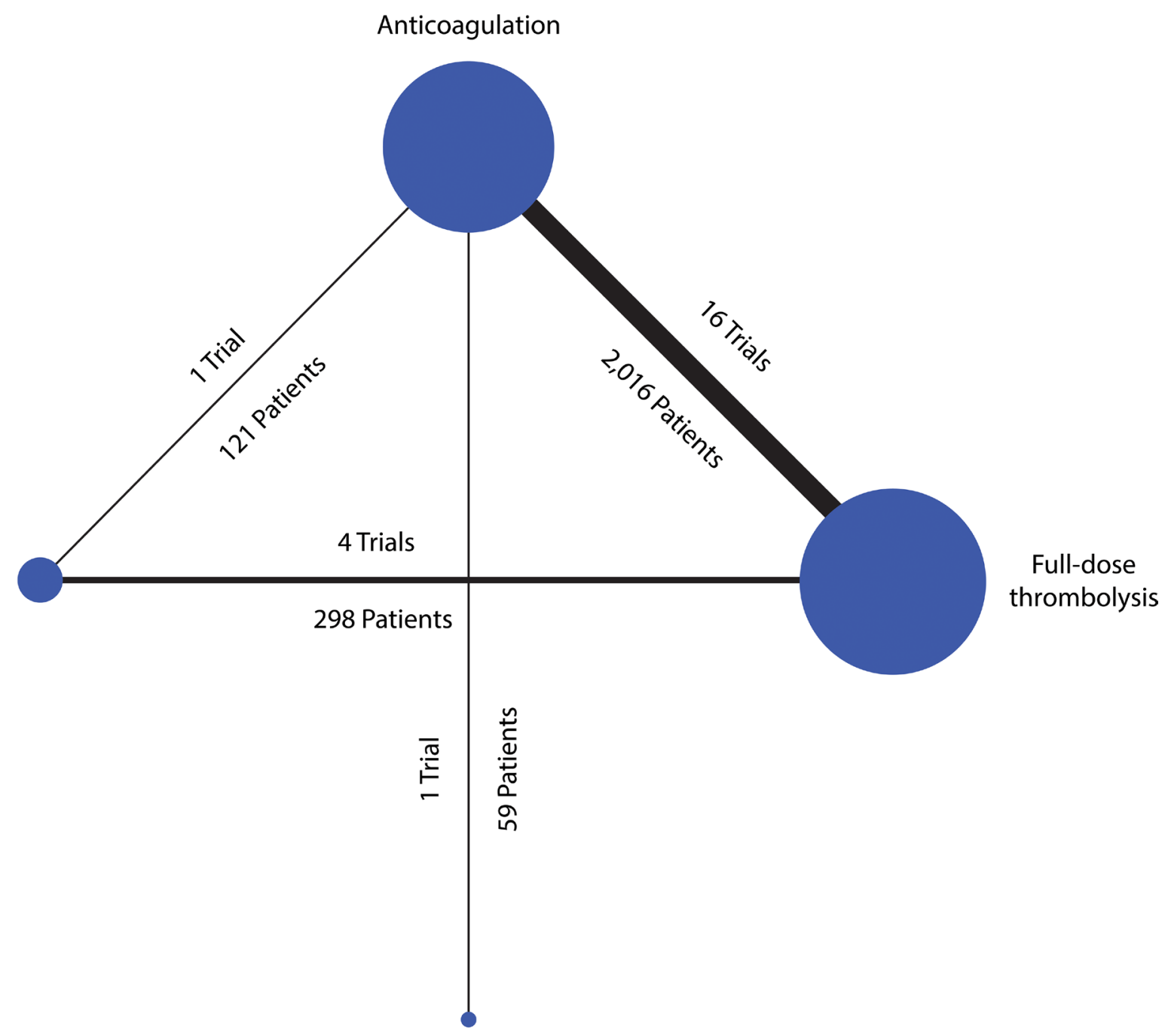

\section{Catheter-directed thrombolysis}

and 2014 and included 2494 participants. The mean study sample size was 113 participants, ranging from 8 to 1005 patients. The baseline characteristics of patients included in these trials are described in table 1 . The primary outcome (all-cause mortality) was reported in all studies.

\section{Direct meta-analysis}

Results of direct pairwise meta-analysis are summarised in table 2 and online supplementary efigures 5-8. All interventions were associated with a non-significant reduction of all-cause mortality (full-dose thrombolysis: OR 0.64; 95\% CI 0.37 to 1.09 ; low-dose thrombolysis: $0.32,0.03$ to 3.13 ; catheter-directed thrombolysis: $0.31,0.01$ to 7.96 ); full-dose thrombolysis was not superior to low-dose thrombolysis $(1.04,0.24$ to 4.41$)$. Full-dose thrombolytic therapy was significantly associated with a greater risk of major bleeding $(2.39,1.44$ to 3.95$)$ and $\mathrm{ICH}(3.66,1.13$ to 11.86) compared with anticoagulant therapy (online supplementary efigures 6 and 7), whereas low-dose thrombolysis showed a non-significant benefit in terms of major bleeding and $\mathrm{ICH}$ compared with full-dose thrombolysis (table 2). All outcomes were associated with negligible heterogeneity $\left(\mathrm{I}^{2}<12 \%\right)$.

\section{Network meta-analysis: primary outcomes}

In network meta-analysis, compared with anticoagulation alone, full-dose thrombolysis was associated with an OR of $0.60(95 \%$ CI 0.36 to 1.01 ), low-dose thrombolysis with an OR of 0.47 (95\% CI 0.14 to 1.59 ) and catheter-directed thrombolysis with an OR of 0.31 (95\% CI 0.01 to 7.96) for dying (figure 2). When recanalisation treatments were compared, none of comparisons reached conventional level of statistical significance (figure 2). In network meta-analysis, compared with anticoagulation alone, full-dose thrombolysis was associated with an OR of $2.00(95 \%$ CI 1.06 to 3.78), low-dose thrombolysis with an OR of 0.90 (95\% CI 0.25 to 3.21) and catheter-directed thrombolysis with an OR of 0.97 (95\% CI 0.02 to 56.03) for bleeding (figure 2). Again, when recanalisation treatments were compared for bleeding, none of comparisons reached conventional level of statistical significance (figure 2).

Network meta-analysis suggested that catheter-directed thrombolysis was associated with the lowest probability of dying (SUCRA, 0.67), followed by low-dose thrombolysis (SUCRA, 0.66) and full-dose thrombolysis (SUCRA, 0.55) (figure 3). Similarly, low-dose thrombolysis was associated with the lowest 


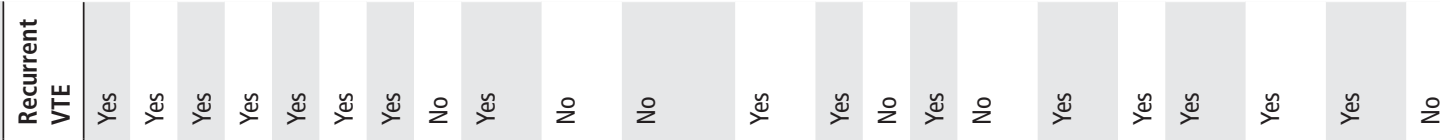

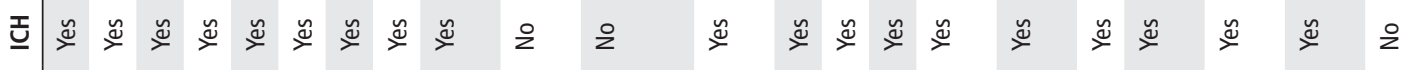

흥

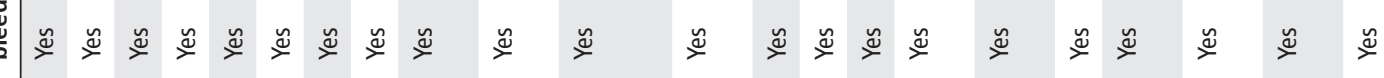

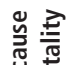

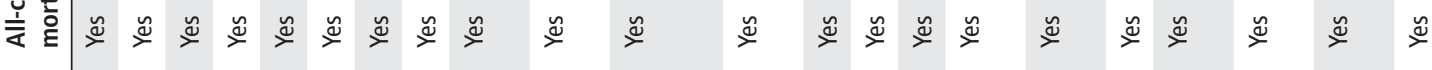

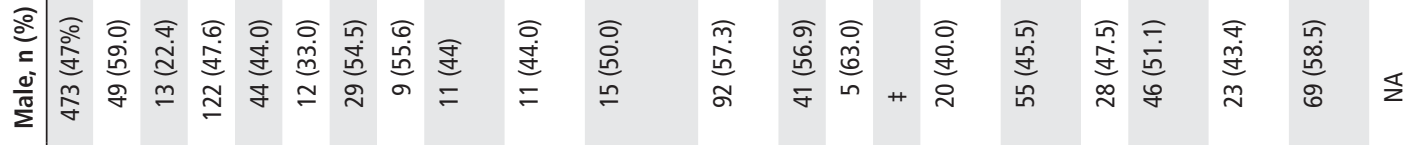

章

ㅎ

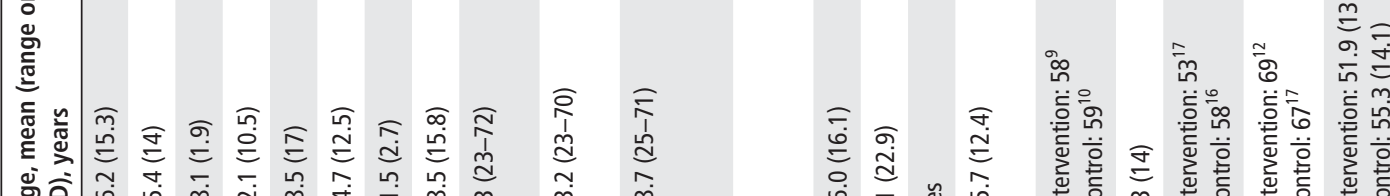

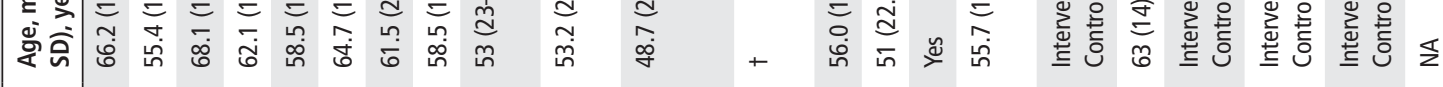

뜸

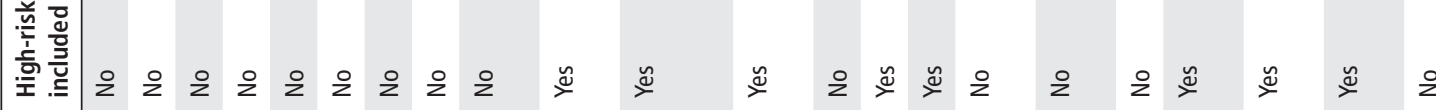

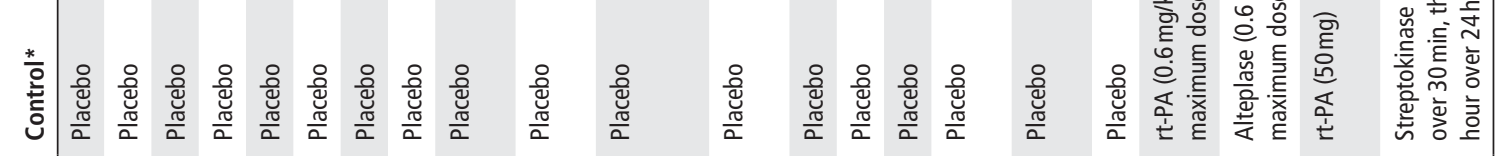

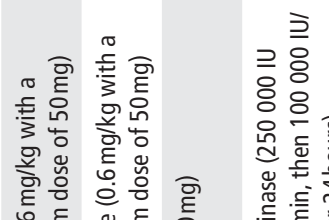

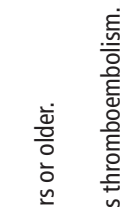


Table 2 Summary of direct meta-analysis for all-cause mortality and adverse event outcomes

\begin{tabular}{|c|c|c|c|c|c|c|}
\hline \multirow[b]{2}{*}{ Intervention } & \multirow[b]{2}{*}{ Studies (n) } & \multicolumn{2}{|c|}{ Active intervention* } & \multicolumn{2}{|c|}{$\begin{array}{l}\text { Control (placebo unless otherwise } \\
\text { noted)* }\end{array}$} & \multirow[b]{2}{*}{ OR $(95 \% \mathrm{CI})$} \\
\hline & & No. with event & Total (n) & No. with event & Total (n) & \\
\hline \multicolumn{7}{|l|}{ All-cause mortality } \\
\hline Full-dose thrombolysis & 16 & 23 & 1010 & 42 & 1006 & $0.64(0.37$ to 1.09$)$ \\
\hline Low-dose thrombolysis & 1 & 1 & 61 & 3 & 60 & 0.32 (0.03 to 3.13$)$ \\
\hline Catheter-directed thrombolysis & 1 & 0 & 30 & 1 & 29 & $0.31(0.01$ to 7.96$)$ \\
\hline $\begin{array}{l}\text { Full-dose thrombolysis versus low-dose } \\
\text { thrombolysis }\end{array}$ & 4 & 4 & 112 & 7 & 186 & 1.04 (0.24 to 4.41$)$ \\
\hline \multicolumn{7}{|l|}{ Major bleeding } \\
\hline Full-dose thrombolysis & 16 & 99 & 1010 & 38 & 1006 & 2.39 (1.44 to 3.95$)$ \\
\hline Low-dose thrombolysis & 1 & 0 & 61 & 0 & 60 & Not estimable \\
\hline Catheter-directed thrombolysis & 1 & 0 & 30 & 0 & 29 & Not estimable \\
\hline $\begin{array}{l}\text { Full-dose thrombolysis versus low-dose } \\
\text { thrombolysis }\end{array}$ & 4 & 9 & 112 & 7 & 186 & 2.26 (0.78 to 6.58$)$ \\
\hline \multicolumn{7}{|l|}{ Intracranial haemorrhage } \\
\hline Full-dose thrombolysis & $\begin{array}{r}14 \\
2\end{array}$ & 15 & 983 & 2 & 978 & 3.66 (1.13 to 11.86$)$ \\
\hline Low-dose thrombolysis & 1 & 0 & 61 & 0 & 60 & Not estimable \\
\hline Catheter-directed thrombolysis & 1 & 0 & 30 & 0 & 29 & Not estimable \\
\hline $\begin{array}{l}\text { Full-dose thrombolysis versus low-dose } \\
\text { thrombolysis }\end{array}$ & 3 & 3 & 97 & 0 & 161 & 6.85 (0.74 to 63.24$)$ \\
\hline \multicolumn{7}{|l|}{ Recurrent VTE } \\
\hline Full-dose thrombolysis & 11 & 19 & 945 & 37 & 945 & $0.57(0.32$ to 1.03$)$ \\
\hline Low-dose thrombolysis & 1 & 0 & 61 & 3 & 60 & $0.13(0.01$ to 2.64$)$ \\
\hline Catheter-directed thrombolysis & 1 & 0 & 30 & 0 & 29 & Not estimable \\
\hline $\begin{array}{l}\text { Full-dose thrombolysis versus low-dose } \\
\text { thrombolysis }\end{array}$ & 3 & 4 & 97 & 6 & 161 & $1.35(0.36$ to 5.00$)$ \\
\hline
\end{tabular}

probability of major bleeding (SUCRA, 0.61), followed by catheter-directed thrombolysis (SUCRA, 0.54) and full-dose thrombolysis (SUCRA, 0.17) (figure 3).
Network meta-analysis: secondary outcomes

In network meta-analysis, compared with anticoagulation, all procedures had 0.48-2.07 odds of being associated with $\mathrm{ICH}$ (online supplementary efigure 9). Compared with anticoagulant therapy, low-dose thrombolysis was associated with the lowest

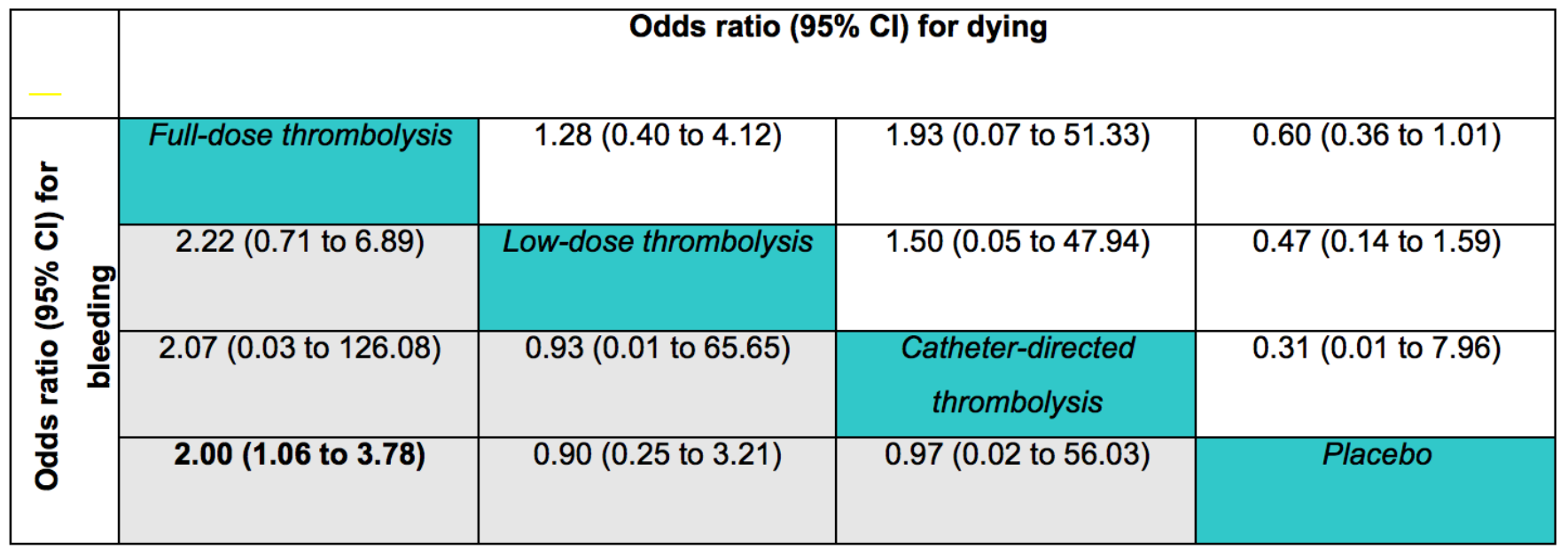

Figure 2 Network meta-analysis estimates of all-cause mortality (upper triangle) and major bleeding (lower triangle) for each comparison. 


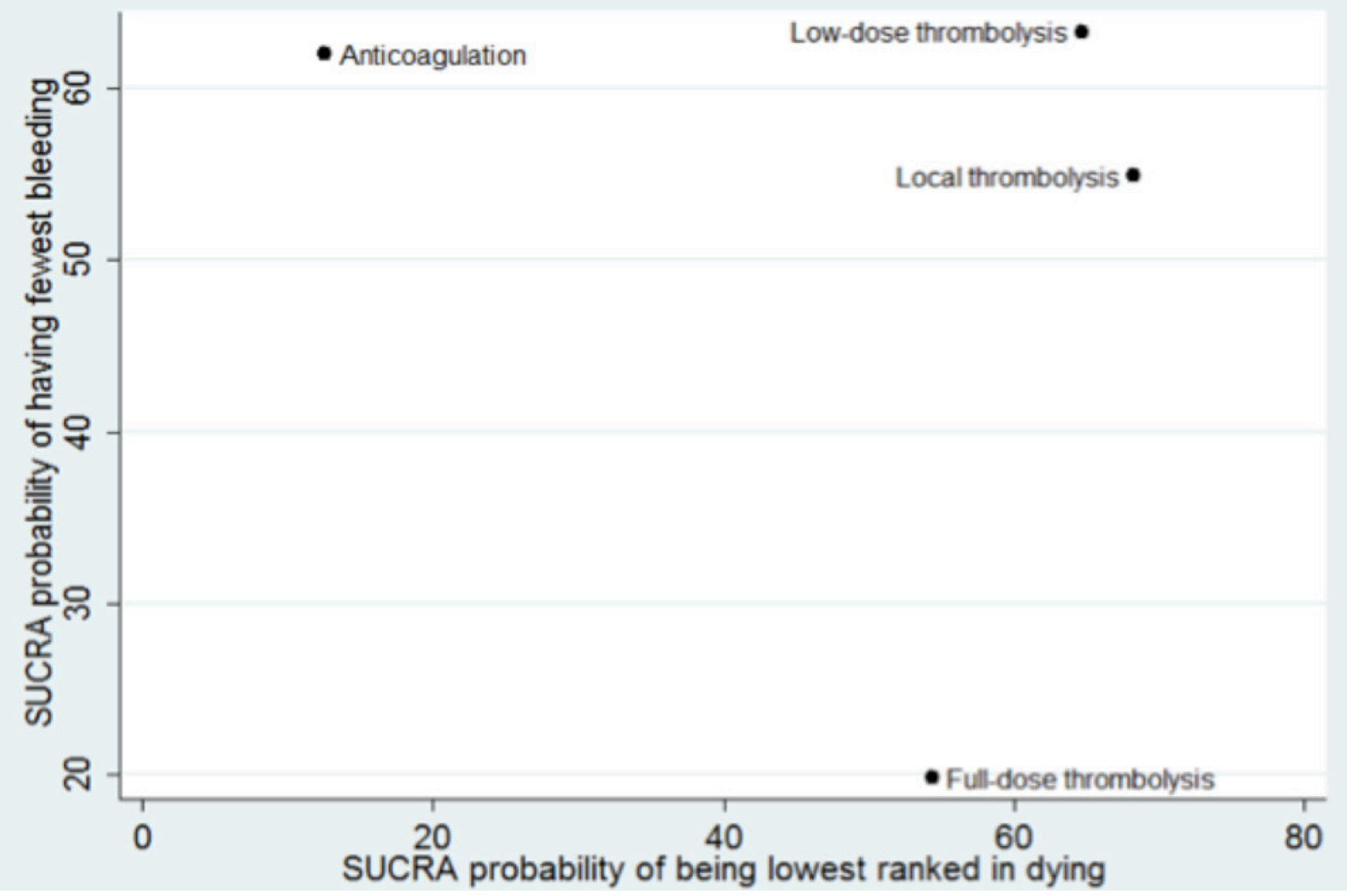

Figure 3 Clustered ranking plot based on cluster analysis of SUCRA for benefit (all-cause mortality) and safety (major bleeding). Treatments lying in the upper right corner are more effective and safe than the other treatments. SUCRA, surface under the cumulative ranking curve.

odds of ICH (OR 0.48; 95\% CI 0.07 to 3.14; SUCRA, 0.78), whereas full-dose thrombolysis (OR 2.07; 95\% CI 0.86 to 5.02 ; SUCRA, 0.16) was associated with the highest odds of $\mathrm{ICH}$ (online supplementary efigure 10).

Compared with anticoagulation, all procedures had 0.34-0.97 lower odds of being associated with recurrent embolism (online supplementary efigure 9). Compared with anticoagulant therapy, low-dose thrombolysis was associated with the lowest odds of recurrent embolism (OR 0.34; 95\% CI 0.09 to 1.25; SUCRA, 0.81 ), whereas catheter-directed thrombolysis (OR 0.97; 95\% CI 0.02 to 50.36; SUCRA, 0.40) was associated with the highest odds of recurrent embolism (online supplementary efigure 10).

\section{Sensitivity analysis}

Results from sensitivity analyses are reported in online supplementary etable 1 . Overall, the results were similar to the main analysis for the primary outcome in sensitivity analyses based on (1) restricting only to studies in patients with haemodynamically stable PE; (2) restricting only to trials where the mean age of participants in the thrombolytic group was $>65$ years; and (3) alternative statistical model (frequentist approach using a random-effects inconsistency model).

\section{Publication bias and network consistency}

There was no evidence of publication bias, either qualitatively based on funnel-plot asymmetry (online supplementary efigure 11) or quantitatively (Egger regression test, $P>0.05$ for all comparisons), although the number of studies included in each comparison was small. There were significant differences between direct and indirect estimates in the only closed loop that allowed assessment of network consistency (anticoagulation-full-dose thrombolysis-low-dose thrombolysis).

\section{Quality of evidence}

The risk of bias summary and figure for included studies are listed in online supplementary efigure 12 and etable 2. Some studies did not present details for randomisation, allocation concealment and blinding. No more than four of the included trials $(<20 \%)$ were deemed to be at high risk of bias in only three domains (randomisation, allocation concealment, blinding) of the Cochrane Collaboration risk of bias tool. In most domains, the majority of trials were at low risk, except for the allocation concealment and blinding categories in which most trials were at an unclear risk due to inadequate reporting of methods.

\section{DISCUSSION}

To our knowledge, this is the first network meta-analysis comparing full-dose thrombolysis, low-dose thrombolysis, catheter-directed thrombolysis and inactive controls on mortality and other adverse outcomes in patients with acute symptomatic PE. The study has several key findings. First, full-dose thrombolysis, low-dose thrombolysis and catheter-directed thrombolysis showed a non-significant trend toward lower risk of all-cause death compared with anticoagulation. Second, fulldose thrombolysis was associated with higher odds of major bleeding compared with anticoagulant treatment, with moderate 
confidence in estimates, but was associated with lower odds of recurrences. Third, low-dose thrombolysis was the treatment that performed best in terms of efficacy (all-cause mortality) and safety (major bleeding). However, the clinical interpretation of these findings is limited by the uncertainty around these estimates and by the potential bias due to the small number of trials in each node.

Traditional pairwise meta-analyses are limited in helping to summarise the most effective treatment among different kinds of recanalisation procedures. Other than comparisons between fulldose thrombolysis and anticoagulant therapy, ${ }^{37} 38$ the number of studies that analysed each particular pair of treatments is still relatively small. Furthermore, for some procedures (ie, fulldose vs catheter-directed thrombolysis), there was no direct comparative research. The ability to estimate effectiveness in this work using network meta-analysis allows for more comprehensive assessment of treatment options than has been previously possible. Additionally, in contrast to separate pairwise analyses, we have been able to rank each treatment based on the strength of its association with mortality and bleeding. Even though the results of the pairwise and network meta-analyses were mostly similar, the biggest difference was seen in the comparison of fulldose with anticoagulation on ICH with the pairwise meta-analysis estimating a larger association than the network model. This was most likely because of the large amount of between-study variation observed in the indirect comparisons being incorporated into the analysis.

Some previous pairwise meta-analyses showed significantly lower associated mortality with full-dose systemic thrombolytic use in PE. ${ }^{37}{ }^{38}$ In our study, we did not find a significant reduction in all-cause mortality with full-dose thrombolytic therapy. This discrepancy between the studies may be explained at least in part by the use of different methodological and statistical techniques. For low-dose systemic thrombolysis and catheter-directed thrombolysis, lack of statistical power might account for the non-significant results, as suggested by the wider CIs. Alternatively, full-dose systemic thrombolysis showed a significant association with major bleeding, a finding consistent with previous meta-analyses. ${ }^{37-39}$ While low-dose and catheter-directed thrombolysis have the potential to offer benefits of full-dose systemic thrombolysis while minimising bleeding risk attributable to a lower dose of the thrombolytic agent, limited randomised clinical trial data might be the main obstacle for providing a definitive conclusion on the comparison of the effect of different reperfusion therapies on major bleeding and $\mathrm{ICH}$. On balance, our results show that low-dose and catheter-directed thrombolysis seem the most highly ranked treatment across the two primary outcomes. Since catheter-directed thrombolysis requires rapid access to the cardiac catheterisation or interventional radiology laboratory, ${ }^{5}$ low-dose thrombolysis is appealing for patients with PE when early recanalisation procedures are indicated. However, it should be kept in mind that, in patients with such presentations, particularly when PE is associated with haemodynamic instability, there are relatively few data for any approaches other than standard-dose systemic thrombolysis.

Ultimately, given the differences in safety, efficacy, and response to therapy, from a clinical perspective, the clinician should always consider the overall clinical picture, and patient management plans need to balance the risks and benefits. There is also a need for randomised trials that compare low-dose thrombolytic therapy with anticoagulation alone in stable patients who have intermediate-risk to high-risk PE. Evidence from such studies would place the role of this procedure for PE on a firmer footing.
This study has limitations. First, there was a paucity of headto-head trials. Second, the biggest threat to validity of the results of any meta-analysis is conceptual heterogeneity (ie, considerable differences among trials in patient characteristics, studied interventions, outcome assessment or study design), which can limit the comparability of trials. Strategies to limit the effect of conceptual heterogeneity included strict inclusion and exclusion criteria and the use of various sensitivity analyses to assess the robustness of the results. Third, we found inconsistency for efficacy, which was mainly determined by the loop of anticoagulation-full-dose thrombolysis-low-dose thrombolysis. Since some evidence suggests that quality of thrombolytic clinical trials has substantially changed in the past 30 years, we believe that this inconsistency might be a consequence of a cohort effect that relates to different methods used in the older studies compared with those done more recently. ${ }^{40}$ Fourth, ranking probabilities may be affected by unequal numbers of trials per comparison, sample size of individual studies, network configuration and effect sizes among treatments and should be interpreted with caution. Finally, some included trials had an unclear or high rate of selection and performance bias, and there are unaddressed concerns regarding the effect of recanalisation procedures in a clinical setting.

In conclusion, compared with standard anticoagulation, recanalisation procedures had a similar risk of all-cause mortality, though full-dose thrombolysis was associated with an increased risk of major bleeding. This network meta-analysis did not identify a statistically significant difference between the outcomes associated with these therapies, but low-dose thrombolysis was associated with the lowest probability of dying and bleeding. The current body of evidence is limited, and further conclusive studies are needed to establish the role of each of the recanalisation procedures.

\section{Author affiliations}

${ }^{1}$ Respiratory Department, Hospital Ramón y Cajal, Madrid, Spain

${ }^{2}$ Medicine Department, Universidad de Alcala (IRYCIS), Alcalá de Henares, Spain

${ }^{3}$ Faculty of Health Sciences, Universidad Francisco de Vitoria, Pozuelo de Alarcon, Spain

${ }^{4}$ Biostatistics Department, Ramón y Cajal Hospital, IRYCIS, CIBERESP, Madrid, Spain ${ }^{5}$ Department of Thrombosis and Haemostasis, Leiden University Medical Center, Leiden, The Netherlands

${ }^{6}$ Cedars-Sinai Medical Center, Los Angeles, California, USA

${ }^{7}$ Divisions of Pulmonary and Critical Care Medicine and General Medical Sciences, Washington University School of Medicine, St Louis, Missouri, USA

Contributors Study concept and design: DJ, MVH, VT and RDY. Acquisition of data, analysis and interpretation of data and statistical analysis: DJ, CM-S, AM, JZ, RM, DB, FAK, MVH, VT and RDY. Drafting of the manuscript: DJ, FAK, MVH, VT and RDY. Critical revision of the manuscript for important intellectual content: DJ, CM-S, AM, JZ, RM, DB, FAK, MVH, VT and RDY. Study supervision: DJ and RDY. The corresponding author, DJ, had full access to all the data in the study and had final responsibility for the decision to submit for publication.

Funding This study (PII11/00246) was supported by the Instituto de Salud Carlos III (Plan Estatal de I+D+i 2013-2016) and co-financed by the European Development Regional Fund 'A way to achieve Europe'.

Competing interests None declared.

Provenance and peer review Not commissioned; externally peer reviewed.

(c) Article author(s) (or their employer(s) unless otherwise stated in the text of the article) 2018. All rights reserved. No commercial use is permitted unless otherwise expressly granted.

\section{REFERENCES}

1 Goldhaber SZ, Visani L, De Rosa M. Acute pulmonary embolism: clinical outcomes in the International Cooperative Pulmonary Embolism Registry (ICOPER). Lancet 1999;353:1386-9. 
2 Jiménez D, de Miguel-Díez J, Guijarro R, et al. Trends in the management and outcomes of acute pulmonary embolism: analysis from the RIETE registry. J Am Coll Cardiol 2016;67:162-70.

3 Becattini C, Agnelli G. Risk factors for adverse short-term outcome in patients with pulmonary embolism. Thromb Res 2001;103:V239-V244.

4 Conget $F$, Otero $R$, Jiménez $D$, et al. Short-term clinical outcome after acute symptomatic pulmonary embolism. Thromb Haemost 2008; 100:937-42.

5 Kearon C, AkI EA, Ornelas J, et al. Antithrombotic therapy for VTE disease: CHEST guideline expert panel report. Chest 2016;149:315-52.

6 Meyer G, Vicaut E, Danays T, et al. Fibrinolysis for patients with intermediate-risk pulmonary embolism. N Engl J Med 2014;370:1402-11.

7 Konstantinides SV, Torbicki A, Agnelli G, et al. 2014 ESC guidelines on the diagnosis and management of acute pulmonary embolism. Eur Heart J 2014;35:3033-73.

8 Hutton B, Salanti G, Caldwell DM, et al. The PRISMA extension statement for reporting of systematic reviews incorporating network meta-analyses of health care interventions: checklist and explanations. Ann Intern Med 2015;162:777-84.

9 Lefebvre C, Manheimer E, Glanville J. Chapter 6: Searching for studies. In: Higgins JPT, Green S, eds. Cochrane handbook for systematic reviews of interventions. London, England: Cochrane Collaboration, 2011.

10 Cochrane Higgins JPT Green S eds. Cochrane handbook for systematic reviews of interventions [version 5.1.0, updated March 2011]. http://handbook.cochrane.org/ (accessed 20 Jun 2016).

11 DerSimonian R, Laird N. Meta-analysis in clinical trials. Control Clin Trials 1986;7:177-88.

12 Higgins JP, Thompson SG. Quantifying heterogeneity in a meta-analysis. Stat Med 2002;21:1539-58.

13 Caldwell DM, Ades AE, Higgins JP. Simultaneous comparison of multiple treatments: combining direct and indirect evidence. BMJ 2005;331:897-900.

14 White IR, Barrett JK, Jackson D, et al. Consistency and inconsistency in network meta-analysis: concepts and models for multi-arm studies. Res Synth Methods 2012;3:111-25.

15 Salanti G, Ades AE, loannidis JP. Graphical methods and numerical summaries for presenting results from multiple-treatment meta-analysis: an overview and tutorial. J Clin Epidemiol 2011;64:163-71.

16 Kline JA, Nordenholz KE, Courtney DM, et al. Treatment of submassive pulmonary embolism with tenecteplase or placebo: cardiopulmonary outcomes at 3 months: multicenter double-blind, placebo-controlled randomized trial. J Thromb Haemost 2014;12:459-68.

17 Becattini C, Agnelli G, Salvi A, et al. Bolus tenecteplase for right ventricle dysfunction in hemodynamically stable patients with pulmonary embolism. Thromb Res 2010;125:e82-e86.

18 Konstantinides S, Geibel A, Heusel G, et al. Heparin plus alteplase compared with heparin alone in patients with submassive pulmonary embolism. N Engl J Med 2002;347:1143-50

19 Goldhaber SZ, Haire WD, Feldstein ML, et al. Alteplase versus heparin in acute pulmonary embolism: randomised trial assessing right-ventricular function and pulmonary perfusion. Lancet 1993;341:507-11.

20 Dalla-Volta S, Palla A, Santolicandro A, et al. PAIMS 2: alteplase combined with heparin versus heparin in the treatment of acute pulmonary embolism. Plasminogen activator Italian multicenter study 2. J Am Coll Cardiol 1992;20:520-6.

21 Levine $\mathrm{M}$, Hirsh J, Weitz J, et al. A randomized trial of a single bolus dosage regimen of recombinant tissue plasminogen activator in patients with acute pulmonary embolism. Chest 1990;98:1473-9.
22 Investigators P. Tissue plasminogen activator for the treatment of acute pulmonary embolism: a collaborative study by the PIOPED Investigators. Chest 1990;97:528-33.

23 Marini C, Di Ricco G, Rossi G, et al. Fibrinolytic effects of urokinase and heparin in acute pulmonary embolism: a randomized clinical trial. Respiration 1988;54:162-73.

24 Ly B, Arnesen H, Eie H, et al. A controlled clinical trial of streptokinase and heparin in the treatment of major pulmonary embolism. Acta Med Scand 1978;203:465-70.

25 Tibbutt DA, Davies JA, Anderson JA, et al. Comparison by controlled clinical trial of streptokinase and heparin in treatment of life-threatening pulmonay embolism. $\mathrm{Br}$ Med J 1974;1:343-7.

26 The Urokinase Pulmonary Embolism Trial. Urokinase pulmonary embolism trial. Phase 1 results: a cooperative study. JAMA 1970;214:2163-72.

27 Fasullo S, Scalzo S, Maringhini G, et al. Six-month echocardiographic study in patients with submassive pulmonary embolism and right ventricle dysfunction: comparison of thrombolysis with heparin. Am J Med Sci 2011;341:33-9.

28 Jerjes-Sanchez C, Ramírez-Rivera A, de Lourdes García M, et al. Streptokinase and heparin vs heparin alone in massive pulmonary embolism: a randomized controlled trial. J Thromb Thrombolysis 1995;2:227-9.

29 Dotter CT, Seaman AJ, Rösch J, et al. Streptokinase and heparin in the treatment of pulmonary embolism: a randomized comparison. Vasc Surg 1979;13:42-52.

30 Taherkhani M, Taherkhani A, Hashemi SR, et al. Thrombolytic-plus-anticoagulant therapy versus anticoagulant-alone therapy in submassive pulmonary thromboembolism (TVASPE Study): a randomized clinical trial. J Tehran Heart Cent 2014;9:104-8.

31 Sharifi M, Bay C, Skrocki L, et al. Moderate pulmonary embolism treated with thrombolysis (from the "MOPETT" Trial). Am J Cardiol 2013;111:273-7.

32 Kucher N, Boekstegers P, Müller OJ, et al. Randomized, controlled trial of ultrasoundassisted catheter-directed thrombolysis for acute intermediate-risk pulmonary embolism. Circulation 2014;129:479-86.

33 Goldhaber SZ, Agnelli G, Levine MN. Reduced dose bolus alteplase vs conventional alteplase infusion for pulmonary embolism thrombolysis. An international multicenter randomized trial. The Bolus Alteplase Pulmonary Embolism Group. Chest 1994; 106:718-24

34 Sors H, Pacouret G, Azarian R, et al. Hemodynamic effects of bolus vs 2-h infusion of alteplase in acute massive pulmonary embolism. A randomized controlled multicenter trial. Chest 1994;106:712-7.

35 Wang C, Zhai Z, Yang Y, et al. Efficacy and safety of low dose recombinant tissue-type plasminogen activator for the treatment of acute pulmonary thromboembolism: a randomized, multicenter, controlled trial. Chest 2010;137:254-62

36 Abdelsamad AA, El-Morsi AS, Mansour AE. Efficacy and safety of high dose versus low dose streptokinase for treatment of submassive pulmonary embolism. Egy Heart $J$ 2011;63:67-72.

37 Chatterjee S, Chakraborty A, Weinberg I, et al. Thrombolysis for pulmonary embolism and risk of all-cause mortality, major bleeding, and intracranial hemorrhage: a metaanalysis. JAMA 2014;311:2414-21.

38 Marti C, John G, Konstantinides S, et al. Systemic thrombolytic therapy for acute pulmonary embolism: a systematic review and meta-analysis. Eur Heart J 2015;36:605-14

39 Riera-Mestre A, Becattini C, Giustozzi M, et al. Thrombolysis in hemodynamically stable patients with acute pulmonary embolism: a meta-analysis. Thromb Res 2014:134:1265-71.

40 Konstantinides SV. Acute pulmonary embolism revisited. Heart 2008;94:795-802 